\title{
Clustering-Based Symmetric Radial Basis Function Beamforming
}

\author{
S. Chen, K. Labib, and L. Hanzo
}

\begin{abstract}
We propose a clustering-based symmetric radial basis function (SRBF) detector for multiple-antenna assisted beamforming systems. By exploiting the inherent symmetry of the underlying optimal Bayesian detection solution, this SRBF detector is capable of realizing the optimal Bayesian performance by clustering noisy observation data using an enhanced $\kappa$-means clustering algorithm. The proposed adaptive solution provides a signal-to-noise ratio gain in excess of $8 \mathrm{~dB}$ against the theoretical linear minimum bit error rate benchmark, when supporting five users with the aid of three receive antennas.
\end{abstract}

Index Terms-Beamforming, clustering, multiple-antenna system, radial basis function network, symmetry.

\section{INTRODUCTION}

A DAPTIVE beamforming is capable of separating user signals transmitted on the same carrier frequency, and thus provides a practical means of supporting multiusers in a space division multiple access scenario [1]-[7]. Classically, this is achieved by a linear beamformer based on the minimum mean square error (L-MMSE) solution [1], [5], [6], [8]. The optimal solution for the linear beamforming has been shown to be the minimum bit error rate (L-MBER) design [9] which outperforms the L-MMSE one and is capable of operating in hostile rank-deficient scenarios. However, digital communication signal detection can be viewed as a classification problem [10]-[13], where the receiver detector simply classifies the received multidimensional channel-impaired signal into the most-likely transmitted symbol constellation point or class. This achieves significantly better performance over the linear detection approach at the cost of an increase in detection complexity. We apply this nonlinear detection approach to multiple-antenna assisted beamforming systems and derive the optimal Bayesian nonlinear detection solution. However, directly realizing this optimal Bayesian detector requires the knowledge of the overall system channel matrix, which is very difficult if not impossible to identify, since the receiver does not have access to the interfering users' transmitted data.

We circumvent this difficulty by proposing an adaptive radial basis function (RBF) based solution. We highlight an inherent symmetry property of the underlying optimal Bayesian

Manuscript received October 23, 2006; revised January 25, 2007. The associate editor coordinating the review of this manuscript and approving it for publication was Prof. Zhengdao Wang.

The authors are with the School of Electronics and Computer Science, University of Southampton, Southampton SO17 1BJ, U.K.

Color versions of one or more of the figures in this paper are available online at http://ieeexplore.ieee.org.

Digital Object Identifier 10.1109/LSP.2007.896149 nonlinear detection solution, and this naturally leads to the proposal of a novel symmetric RBF (SRBF) based detector to realize the true optimal nonlinear detection solution. A simple and effective adaptive algorithm is proposed based on the enhanced $\kappa$-means clustering algorithm [14], [15], which is capable of finding the optimal RBF center positions by clustering noisy observation data. This adaptive clustering algorithm is very simple and it is computationally no more complex than the stochastic adaptive algorithm for realising the L-MBER solution, known as the least bit error rate algorithm [9]. The advantage of the proposed SRBF detector is demonstrated in challenging detection scenarios, where the number of users supported is almost twice the number of antenna array elements. The proposed adaptive solution is shown to provide a signal-to-noise ratio (SNR) gain in excess of $8 \mathrm{~dB}$ against the theoretical linear minimum bit error rate benchmark, when supporting five users with the aid of three receive antennas. It is also demonstrated that the performance of the SRBF detector is robust to the choices of the RBF variance value and the detector's size.

\section{Multiple Antenna Assisted BeAmForming}

The system supports $M$ users, where each user transmits on the same carrier frequency. Without the loss of generality, source 1 is assumed to be the desired user and the rest of the sources are the interfering users. For such a system, user separation can be achieved in the spatial or angular domain [6], [7], and the receiver is equipped with a linear antenna array consisting of $L$ uniformly spaced elements. Further assume that the channel is non-dispersive. Then the symbol-rate sampled complex-valued received signal vector $\mathbf{x}(k)=\left[x_{1}(k) x_{2}(k) \cdots x_{L}(k)\right]^{T}$ can be expressed as

$$
\begin{aligned}
\mathbf{x}(k) & =\mathbf{P} \mathbf{b}(k)+\mathbf{n}(k) \\
& =\left[A_{1} \mathbf{s}_{1} A_{2} \mathbf{s}_{2} \cdots A_{M} \mathbf{s}_{M}\right] \mathbf{b}(k)+\mathbf{n}(k) \\
& =\overline{\mathbf{x}}(k)+\mathbf{n}(k)
\end{aligned}
$$

where $A_{i}$ is the nondispersive channel coefficient of user $i, \mathbf{s}_{i}$ is the steering vector of source $i, \mathbf{b}(k)=$ $\left[b_{1}(k) b_{2}(k) \cdots b_{M}(k)\right]^{T}$ is the $k$ th transmitted symbol vector associated with the $M$ users, the $L$-channel noise vector $\mathbf{n}(k)=\left[n_{1}(k) n_{2}(k) \cdots n_{L}(k)\right]^{T}$ is Gaussian with $E\left[\mathbf{n}(k) \mathbf{n}^{H}(k)\right]=2 \sigma_{n}^{2} \mathbf{I}_{L}$, and $\mathbf{I}_{L}$ denotes the $L \times L$ identity matrix. The user data symbol $b_{i}(k)$ takes values from a binary phase shift keying (BPSK) symbol set, i.e. $b_{i}(k) \in\{ \pm 1\}$. The desired user's SNR is given by SNR $=\left|A_{1}\right|^{2} \sigma_{b}^{2} / 2 \sigma_{n}^{2}$, where $\sigma_{b}^{2}=1$ is the BPSK symbol energy, and the desired signal to interferer $i$ ratio is defined by $\mathrm{SIR}_{i}=\left|A_{1}\right|^{2} /\left|A_{i}\right|^{2}$, for $2 \leq i \leq M$. 
Traditionally, a linear beamforming receiver, $y_{\text {Lin }}(k)=$ $\boldsymbol{\theta}^{T} \mathbf{x}(k)$, is adopted to detect the desired user's signal [1], [5] with the associated detector's decision given by

$$
\hat{b}_{1}(k)=\operatorname{sgn}\left(\Re\left[y_{\text {Lin }}(k)\right]\right)= \begin{cases}+1, & \Re\left[y_{\text {Lin }}(k)\right] \geq 0 \\ -1, & \Re\left[y_{\text {Lin }}(k)\right]<0\end{cases}
$$

where $\boldsymbol{\theta}=\left[\theta_{1} \theta_{2} \cdots \theta_{L}\right]^{T}$ denotes the complex-valued linear beamformer's weight vector and $\Re[\bullet]$ the real part. Classically, the L-MMSE solution for the weight vector of the linear beamformer is regarded as the optimal design [1], [5], [6], [8]. The optimal weight vector designed for the linear beamformer in fact is known to be the L-MBER solution [9], which directly minimises the bit error rate (BER) of the linear beamformer and is capable of operating in rank-deficient scenarios. The L-MBER beamformer has a larger user capacity and is significantly more robust to the near-far effects, in comparison with the L-MMSE design [9].

However, the true optimal multiple antenna aided beamforming detector is nonlinear. This is because the optimal detection rule for the generic communication signal detection problem is the maximum a posteriori (MAP) detection [10], which is inherently nonlinear. We now derive this optimal nonlinear solution for beamforming detector. Let us denote the $N_{b}=2^{M}$ legitimate sequences of $\mathbf{b}(k)$ as $\mathbf{b}_{q}, 1 \leq q \leq N_{b}$, and the first element of $\mathbf{b}_{q}$, related to the desired user, as $b_{q, 1}$. The noiseless channel output $\overline{\mathbf{x}}(k)$ takes values from the finite signal set $\overline{\mathbf{x}}(k) \in \mathcal{X} \triangleq\left\{\overline{\mathbf{x}}_{q}=\mathbf{P b}_{q}, 1 \leq q \leq N_{b}\right\}$, which can be divided into two subsets conditioned on the value of $b_{1}(k)$ as

$$
\mathcal{X}^{( \pm)} \triangleq\left\{\overline{\mathbf{x}}_{i} \in \mathcal{X}, 1 \leq i \leq N_{s b}: b_{1}(k)= \pm 1\right\}
$$

where the size of $\mathcal{X}^{(+)}$and $\mathcal{X}^{(-)}$is $N_{s b}=N_{b} / 2=2^{M-1}$. We will assume that all the transmitted data sequences $\mathbf{b}_{q}, 1 \leq q \leq$ $N_{b}$, have the same a priori probability. In this case, the optimal MAP detection becomes the maximum likelihood (ML) detection [10]. Let the conditional probabilities of receiving $\mathbf{x}(k)$ given $b_{1}(k)= \pm 1$ be $p_{ \pm}(\mathbf{x}(k))=p\left(\mathbf{x}(k) \mid b_{1}(k)= \pm 1\right)$. According to Bayes decision theory [16], the optimal detection strategy is

$$
\hat{b}_{1}(k)= \begin{cases}+1, & \text { if } p_{+}(\mathbf{x}(k)) \geq p_{-}(\mathbf{x}(k)) \\ -1, & \text { if } p_{+}(\mathbf{x}(k))<p_{-}(\mathbf{x}(k))\end{cases}
$$

If we introduce the real-valued Bayesian decision variable

$$
y_{\text {Bay }}(k)=f_{\text {Bay }}(\mathbf{x}(k)) \triangleq \frac{1}{2} p_{+}(\mathbf{x}(k))-\frac{1}{2} p_{-}(\mathbf{x}(k))
$$

the optimal detection rule (4) is equivalent to $\hat{b}_{1}(k)=$ $\operatorname{sgn}\left(y_{\text {Bay }}(k)\right)$. From the signal model (1), the decision variable (5) can be expressed as

$$
y_{\text {Bay }}(k)=\sum_{q=1}^{N_{b}} \operatorname{sgn}\left(b_{q, 1}\right) \beta_{q} e^{-\frac{\left\|\mathbf{x}(k)-\overline{\mathbf{x}}_{q}\right\|^{2}}{2 \sigma_{n}^{2}}}
$$

where $\beta_{q}$ denotes the a priori probability of $\overline{\mathbf{x}}_{q}$. Since all the $\overline{\mathbf{x}}_{q}$ are equiprobable, $\beta_{q}=\beta=1 / N_{b}\left(2 \pi \sigma_{n}^{2}\right)^{L}$. We now highlight a symmetric property of this optimal Bayesian detector.

Proposition 1: The two subsets $\mathcal{X}^{(+)}$and $\mathcal{X}^{(-)}$are distributed symmetrically, namely, for any signal state

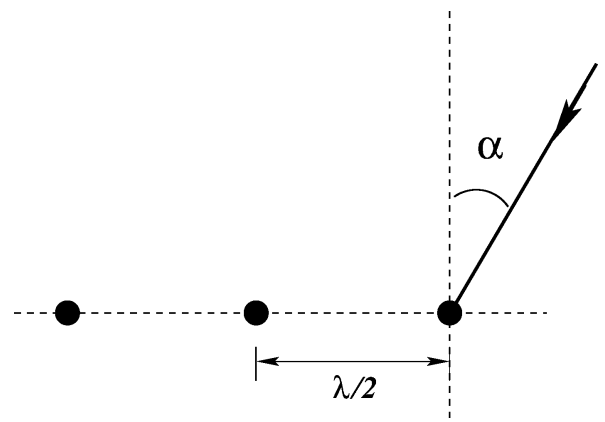

Fig. 1. Geometric structure of the three-element linear array having $\lambda / 2$ spacing, where $\lambda$ is the wavelength and $\alpha$ denotes the angle of arrival of a user.

TABLE I

LOCATIONS OF USERS IN TERMS OF ANGLE OF ARRIVAL (AOA) FOR THE THREE-ELEMENT ANTENNA ARRAY SYSTEM SUPPORTING FIVE USERS

\begin{tabular}{c|ccccc}
\hline \hline user $i$ & 1 & 2 & 3 & 4 & 5 \\
\hline AOA $\alpha$ & $0^{\circ}$ & $10^{\circ}$ & $-17^{\circ}$ & $15^{\circ}$ & $20^{\circ}$ \\
\hline \hline
\end{tabular}

$\overline{\mathbf{x}}_{i}^{(+)} \in \mathcal{X}^{(+)}$there exists a signal state $\overline{\mathbf{x}}_{q}^{(-)} \in \mathcal{X}^{(-)}$satisfying $\overline{\mathbf{x}}_{q}^{(-)}=-\overline{\mathbf{x}}_{i}^{(+)}$.

Proof: Let $\overline{\mathbf{x}}_{i}^{(+)}=\mathbf{P b}_{i}^{(+)} \in \mathcal{X}^{(+)}$. Then $-\overline{\mathbf{x}}_{i}^{(+)}=$ $\mathbf{P}\left(-\mathbf{b}_{i}^{(+)}\right) \in \mathcal{X}^{(-)}$.

Given this symmetry, the optimal Bayesian detector (6) can be characterised as

$$
y_{\text {Bay }}(k)=\sum_{q=1}^{N_{s b}} \beta_{q}\left(e^{-\frac{\left\|\mathbf{x}(k)-\overline{\mathbf{x}}_{q}^{(+)}\right\|^{2}}{2 \sigma_{n}^{2}}}-e^{-\frac{\left\|\mathbf{x}(k)+\overline{\mathbf{x}}_{q}^{(+)}\right\|^{2}}{2 \sigma_{n}^{2}}}\right)
$$

where $\overline{\mathbf{x}}_{q}^{(+)} \in \mathcal{X}^{(+)}$. The Bayesian detector has odd symmetry, as $f_{\text {Bay }}(-\mathbf{x}(k))=-f_{\text {Bay }}(\mathbf{x}(k))$. Although we assume a linear array beamforming structure with BPSK modulation, the approach is actually more general, and it is equally applicable to the narrowband multiple-input multiple-output (MIMO) system modelled by $\mathbf{x}(k)=\mathbf{P b}(k)+\mathbf{n}(k)$, where the $(l, m)$ th element of the channel matrix $\mathbf{P}$ represents the non-dispersive channel connecting the $m$ th transmit antenna to the $l$ th receive antenna. Extension to high-order quadrature amplitute modulation (QAM) schemes is also possible. For example, for the 4-QAM modulation, the noiseless signal state set $\mathcal{X}$ can be divided into the four subsets and the Bayesian decision involves the computation of the four conditional probabilities.

The Bayesian detector requires the channel state set $\mathcal{X}$. If the system matrix $\mathbf{P}$ is known, this state set can be computed. However, the receiver only has access to $b_{1}(k)$, not $\mathbf{b}(k)$, during training. Thus identifying $\mathbf{P}$ is an extremely challenging problem, and it is more realistic for an adaptive scheme to rely only on the noisy observations $\{\mathbf{x}(k)\}$ and the corresponding reference user's data $\left\{b_{1}(k)\right\}$. We proceed to derive such an adaptive nonlinear detector.

\section{Symmetric Radial Basis FunCtion Detector}

The proposed SRBF detector takes the form

$$
y_{\mathrm{SRBF}}(k)=f_{\mathrm{SRBF}}(\mathbf{x}(k))=\sum_{i=1}^{N_{s b}} \theta_{i} \phi_{i}(\mathbf{x}(k))
$$




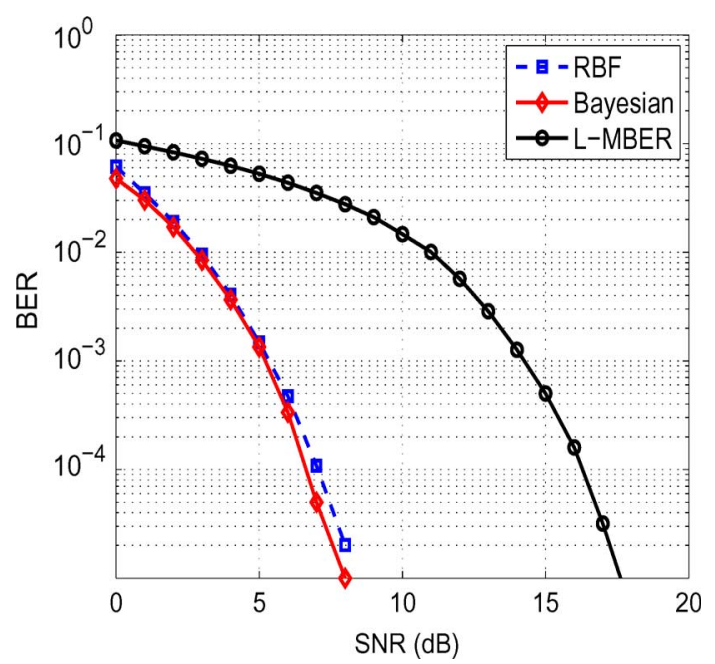

Fig. 2. Desired-user's bit error rate performance for the three-element antenna array supporting five users at the angular positions of Table I. The clusteringbased SRBF detector has $N_{s b}=16$ symmetric RBF nodes.

where $f_{\mathrm{SRBF}}(\bullet)$ is a real-valued nonlinear mapping realized by the SRBF network, $\theta_{i}$ is the $i$ th real-valued RBF weight, $\phi_{i}(\bullet)$ denotes the response of the $i$ th RBF node, and $N_{s b}$ is the number of RBF nodes used. We propose to adopt the following symmetric RBF node

$$
\phi_{i}(\mathbf{x}) \triangleq \varphi\left(\mathbf{x} ; \mathbf{c}_{i}, \sigma_{i}^{2}\right)-\varphi\left(\mathbf{x} ;-\mathbf{c}_{i}, \sigma_{i}^{2}\right)
$$

where $\mathbf{c}_{i}$ is the $i$ th complex-valued RBF center, $\sigma_{i}^{2}$ the $i$ th realvalued $\mathrm{RBF}$ variance, and $\varphi(\bullet)$ the classic $\mathrm{RBF}$ function. In this study, we adopt the Gaussian RBF function

$$
\varphi\left(\mathbf{x} ; \mathbf{c}_{i}, \sigma^{2}\right)=e^{-\frac{\left\|\mathbf{x}-\mathbf{c}_{i}\right\|^{2}}{2 \sigma^{2}}} .
$$

The SRBF network (8) with the node structure (9) has an inherently odd symmetry. A standard RBF model with the RBF node defined by $\phi_{i}(\mathbf{x}) \triangleq \varphi\left(\mathbf{x} ; \mathbf{c}_{i}, \sigma_{i}^{2}\right)$, by contrast, cannot guarantee odd symmetry, particularly when the RBF centers are generated from noisy training data.

It is obvious that we can set all the RBF weights $\theta_{i}=\theta>0$ and all the RBF variances $\sigma_{i}^{2}=\hat{\sigma}_{n}^{2}$, where $\hat{\sigma}_{n}^{2}$ is an estimated $\sigma_{n}^{2}$. Furthermore, if we use the set of signal states $\mathcal{X}^{(+)}$as the RBF centers of this SRBF detector, then it realizes exactly the optimal Bayesian performance. As mentioned previously, however, we can only obtain the RBF centers based on the noisy observations $\{\mathbf{x}(k)\}$, possibly with the aid of the reference user's data $\left\{b_{1}(k)\right\}$. We propose to modify the enhanced $\kappa$-means clustering algorithm [14], [15] to perform this task, which is summarized as follows. During training, the following clustering algorithm is used to update the RBF centers

$$
\mathbf{c}_{i}(k)=\mathbf{c}_{i}(k-1)+\mu_{c} \mathcal{M}_{i}(\check{\mathbf{x}}(k))\left(\check{\mathbf{x}}(k)-\mathbf{c}_{i}(k-1)\right)
$$

where

$$
\check{\mathbf{x}}(k)= \begin{cases}\mathbf{x}(k), & \text { if } b_{1}(k)=+1 \\ -\mathbf{x}(k), & \text { if } b_{1}(k)=-1\end{cases}
$$

and $\mu_{c}$ is the step size. The membership function $\mathcal{M}_{i}(\mathbf{x})$ is defined as

$$
\mathcal{M}_{i}(\mathbf{x})= \begin{cases}1, & \text { if } \bar{v}_{i}\left\|\mathbf{x}-\mathbf{c}_{i}\right\|^{2} \leq \bar{v}_{j}\left\|\mathbf{x}-\mathbf{c}_{j}\right\|^{2}, \forall j \neq i \\ 0, & \text { otherwise }\end{cases}
$$

where $\bar{v}_{i}$ is the variation of the $i$ th cluster. In order to estimate the associated variation $\bar{v}_{i}$, the following updating rule is used:

$\bar{v}_{i}(k)=\mu_{v} \bar{v}_{i}(k-1)+\left(1-\mu_{v}\right) \mathcal{M}_{i}(\check{\mathbf{x}}(k))\left\|\check{\mathbf{x}}(k)-\mathbf{c}_{i}(k-1)\right\|^{2}$

where $\mu_{v}$ is a constant slightly less than 1.0 , e.g. $\mu_{v}=0.995$. The initial variations $\bar{v}_{i}(0), \forall i$, are set to the same small positive number.

The enhanced $\kappa$-means clustering algorithm is guaranteed to converge to the optimal center configuration if the learning rate $\mu_{c}$ is self-adjusting based on an entropy formula or it is fixed to a positive constant that is not to large [14]. Note that the optimal center configuration in our case is the signal state set $\mathcal{X}^{(+)}$. During data transmission, the detector's decision $\hat{b}_{1}(k)$ can be used to substitute $b_{1}(k)$ in (12). The estimated noise variance $\hat{\sigma}_{n}^{2}$ is used as the RBF variance. The performance of the SRBF detector is not overly sensitive to the value of the RBF variance used, and it is known that there exists a large range of the RBF variance values which enable the SRBF detector to achieve the optimal Bayesian performance. This will also be demonstrated in the simulation study. This robustness to the value of the RBF variance is a consequence of the Bayesian detector's robustness to the noise variance $\sigma_{n}^{2}$ used. It has been shown [13] that the performance of the Bayesian detectors using $0.2 \sigma_{n}^{2}$ and $5 \sigma_{n}^{2}$ to substitute the noise variance $\sigma_{n}^{2}$ is indistinguishable from that of the exact Bayesian solution. The true SRBF model size $N_{s b}$ is generally unknown. The influence of the SRBF model size to its performance will be investigated in the simulation study.

\section{Simulation Study}

The simulated beamforming system consisted of three-element linear antenna array and supported $M=5$ users. Fig. 1 shows the array geometric structure and Table I lists the locations of the five users with respect to the antenna array. The simulated narrowband channels were $A_{i}=1+j 0,1 \leq i \leq 5$, and the desired user and all the interfering users had an equal signal power. Therefore, $\mathrm{SIR}_{i}=0 \mathrm{~dB}$ for $2 \leq i \leq 5$. The BER performance of the two benchmarks, the L-MBER beamformer and the Bayesian detector, are shown in Fig. 2. The size of the Bayesian detector is $N_{s b}=16$. Therefore we chose a 16-center SRBF detector with the RBF variance set to $\sigma_{n}^{2}$. The first $N_{s b}$ data points were used as the initial centers $\mathbf{c}_{i}(0)$. The convergence of the proposed clustering algorithm was assessed based on the following Euclidean distance metric:

$$
\operatorname{ED}(k)=\sum_{i=1}^{N_{s b}}\left\|\mathbf{c}_{i}(k)-\overline{\mathbf{x}}_{i}^{(+)}\right\|^{2} .
$$

The initial clustering variations $\bar{v}_{i}(0)$ were set to 0.01 and the adaptive gain for updating the clustering variations was set to $\mu_{v}=0.995$. Fig. 3 depicts the learning curves of the clustering algorithm averaged over five runs for three different values of 


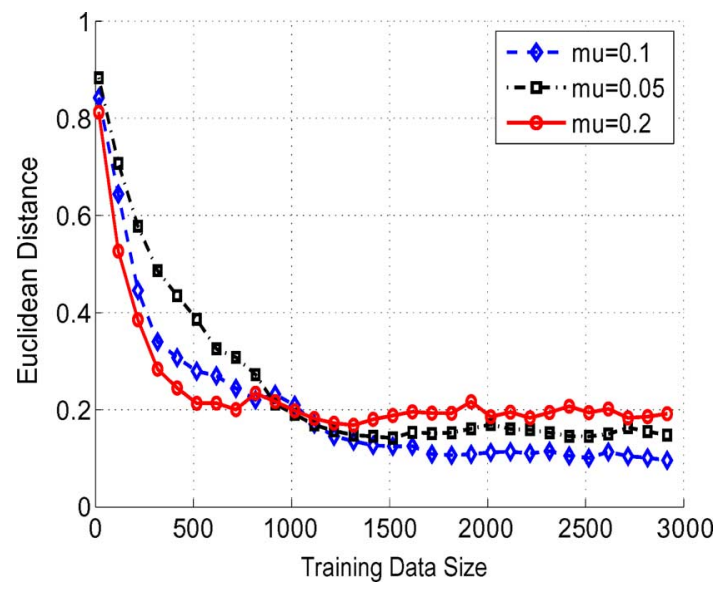

Fig. 3. Learning curve of the clustering-based SRBF detector averaged over 5 runs for the three-element antenna array supporting five users at the angular positions of Table I, where SNR $=5 \mathrm{~dB}$ and the SRBF detector has $N_{s b}=16$ symmetric RBF nodes.

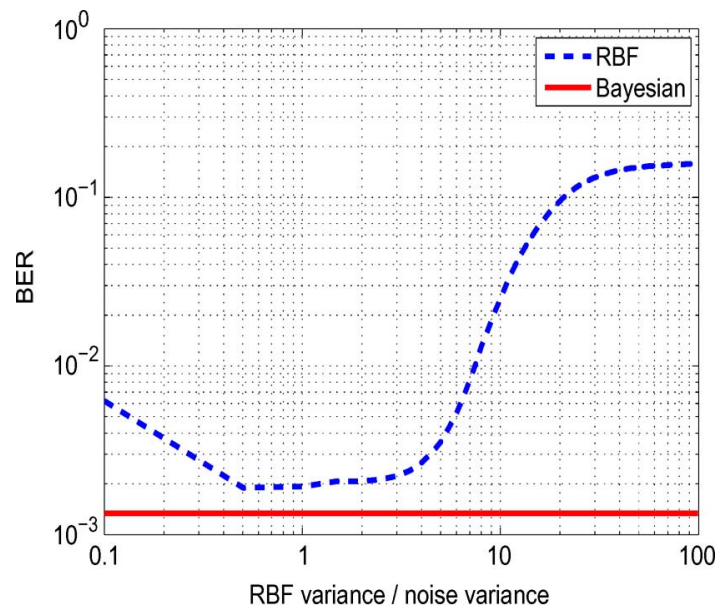

Fig. 4. Influence of the RBF variance to the bit error rate performance of the SRBF detector for the three-element antenna array supporting five users at the angular positions of Table I, where SNR $=5 \mathrm{~dB}$ and the SRBF detector has $N_{s b}=16$ symmetric RBF nodes.

the step size $\mu_{c}$, given SNR $=5 \mathrm{~dB}$. The BER of the clustering-based SRBF detector is also plotted in Fig. 2, in comparison with the optimal Bayesian detector and the theoretical L-MBER detector. We also studied the influence of the RBF variance. For the same conditions in Fig. 3, it was observed that the SRBF detector approached the Bayesian performance given the RBF variance in the range of $0.4 \times \sigma_{n}^{2}$ to $4 \times \sigma_{n}^{2}$, as can be observed in Fig. 4. This confirms that the performance of the SRBF detector is robust to the value of its RBF variance. The influence of the SRBF model size to the detector's performance was next investigated. Given SNR $=5 \mathrm{~dB}$, the BER performance of the clustering-based SRBF detector with different number of RBF nodes after convergence are depicted in Fig. 5.

\section{CONCLUSIONS}

A SRBF network has been proposed for nonlinear detection, which substantially outperforms previous solutions found in the literature in the challenging scenario of supporting almost twice as many users, as the number of antenna elements in multiple-antenna aided beamforming systems. An adaptive algorithm based on the enhanced $\kappa$-means clustering algo-

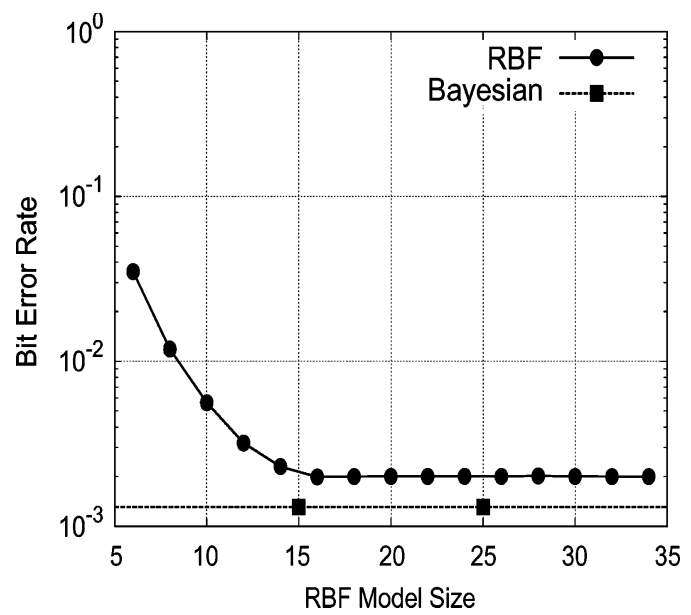

Fig. 5. Influence of the SRBF model size to its bit error rate performance for the three-element antenna array supporting five users at the angular positions of Table I, where SNR $=5 \mathrm{~dB}$.

rithm has been derived for training the SRBF detector. It has been shown that this adaptive SRBF detector is capable of approaching the optimal Bayesian detection performance by clustering noisy observations. The proposed adaptive solution provides an SNR gain in excess of $8 \mathrm{~dB}$ against the powerful linear minimum bit error rate benchmark, when supporting five users with the aid of three receive antennas.

\section{REFERENCES}

[1] J. Litva and T. K. Y. Lo, Digital Beamforming in Wireless Communications. London, U.K.: Artech House, 1996

[2] J. H. Winters, "Smart antennas for wireless systems," IEEE Personal Commun., vol. 5, no. 1, pp. 23-27, 1998.

[3] R. Kohno, "Spatial and temporal communication theory using adaptive antenna array," IEEE Personal Commun., vol. 5, no. 1, pp. 28-35, 1998.

[4] P. Vandenameele, L. van Der Perre, and M. Engels, Space Division Multiple Access for Wireless Local Area Networks. Boston, MA: Kluwer, 2001.

[5] J. S. Blogh and L. Hanzo, Third Generation Systems and Intelligent Wireless Networking-Smart Antennas and Adaptive Modulation. Chichester, U.K.: Wiley, 2002.

[6] A. Paulraj, R. Nabar, and D. Gore, Introduction to Space-Time Wireless Communications. Cambridge, U. K.: Cambridge Univ, Press, 2003.

[7] D. Tse and P. Viswanath, Fundamentals of Wireless Communication. Cambridge, U. K.: Cambridge Univ, Press, 2005.

[8] D. N. C. Tse and S. V. Hanly, "Linear multiuser receivers: Effective interference, effective bandwidth and user capacity," IEEE Trans. Inform, Theory, vol. 45, no. 2, pp. 641-657, Mar. 1999.

[9] S. Chen, N. N. Ahmad, and L. Hanzo, "Adaptive minimum bit error rate beamforming," IEEE Trans. Wireless Commun, vol. 4, no. 2, pp. 341-348, Mar. 2005.

[10] K. Abend and B. D. Fritchman, "Statistical detection for communication channels with intersymbol interference," Proc. IEEE, vol. 58, no. 5, pp. 779-785, May 1970.

[11] S. Chen and B. Mulgrew, "Overcoming co-channel interference using an adaptive radial basis function equaliser," Signal Process., vol. 28, no. 1, pp. 91-107, 1992.

[12] S. Chen, B. Mulgrew, and P. M. Grant, "A clustering technique for digital communications channel equalization using radial basis function networks," IEEE Trans. Neural Netw., vol. 4, no. 4, pp. 570-579, 1993.

[13] S. Chen, S. McLaughlin, B. Mulgrew, and P. M. Grant, "Adaptive Bayesian decision feedback equaliser for dispersive mobile radio channels," IEEE Trans. Commun., vol. 43, no. 5, pp. 1937-1946, May 1995.

[14] C. Chinrungrueng and C. H. Séquin, "Optimal adaptive $\kappa$-means algorithm with dynamic adjustment of learning rate," IEEE Trans. Neural Netw., vol. 6, no. 1, pp. 1873-1896, 1995.

[15] S. Chen, "Nonlinear time series modelling and prediction using Gaussian RBF networks with enhanced clustering and RLS learning," Electron. Lett., vol. 31, no. 2, pp. 117-118, 1995.

[16] R. O. Duda and P. E. Hart, Pattern Classification and Scene Analysis. New York: Wiley, 1973 